\title{
Evaluating the Gradient of the Thin Wire Kernel
}

\author{
Donald R. Wilton $^{(1)}$ and Nathan J. Champagne ${ }^{(2)}$ \\ (1) University of Houston, Houston, TX 77204-4005, USA \\ (2) ESCG, Houston, TX 77258-8477, USA
}

\section{Introduction}

Recently, a formulation for evaluating the thin wire kernel was developed that employed a change of variable to smooth the kernel integrand, canceling the singularity in the integrand [1]. Hence, the typical expansion of the wire kernel in a series for use in the potential integrals is avoided. The new expression for the kernel is exact and may be used directly to determine the gradient of the wire kernel, which consists of components that are parallel and radial to the wire axis.

\section{Integration of the Gradient of the Wire Kernel}

From [1], the thin wire kernel may be written as

$$
K\left(\rho, z-z^{\prime}\right)=\frac{K(\beta)}{2 \pi^{2} R_{\max }} \int_{0}^{1} e^{-j k R_{\max } \operatorname{dn}[\xi K(\beta)]} d \xi,
$$

where $K(\beta)$ is the elliptical integral of the first kind, $z$ is the observation point, $z^{\prime}$ is the source point on the wire, $a$ is the wire radius, $\rho$ is the perpedicular distance from the wire axis to the observation point,

$$
R_{\max }=\sqrt{\left(z-z^{\prime}\right)^{2}+(\rho+a)^{2}} \quad \text { and } \quad \beta^{2}=\frac{4 \rho a}{\left(z-z^{\prime}\right)^{2}+(\rho+a)^{2}}=\frac{4 \rho a}{R_{\max }^{2}} .
$$

Next, the gradient of (1) is written as

$$
\begin{aligned}
\nabla K\left(\rho, z-z^{\prime}\right)= & \nabla\left(\frac{K(\beta)}{2 \pi^{2} R_{\max }} \int_{0}^{1} e^{-j k R_{\max } \operatorname{dn}[\xi K(\beta)]} d \xi\right) \\
= & \left(\frac{\nabla K(\beta)}{K(\beta)}-\frac{\nabla R_{\max }}{R_{\text {max }}}\right)\left(\frac{K(\beta)}{2 \pi^{2} R_{\max }} \int_{0}^{1} e^{-j k R_{\max } \operatorname{dn}[\xi K(\beta)]} d \xi\right) \\
& -j k \frac{K(\beta)}{2 \pi^{2} R_{\text {max }}} \int_{0}^{1} e^{-j k R_{\max } \operatorname{dn}[\xi K(\beta)]} \nabla\left\{R_{\max } \operatorname{dn}[\xi K(\beta)]\right\} d \xi .
\end{aligned}
$$

Substituting

$$
\begin{aligned}
\nabla\left\{R_{\max } \operatorname{dn}[\xi K(\beta)]\right\}= & \operatorname{dn}[\xi K(\beta)] \nabla R_{\max } \\
& -\beta^{2} R_{\max } \xi \operatorname{sn}[\xi K(\beta)] \operatorname{cn}[\xi K(\beta)] \nabla K(\beta)
\end{aligned}
$$

into (3), yields

$$
\begin{aligned}
\nabla K\left(\rho, z-z^{\prime}\right)= & \left(\frac{\nabla K(\beta)}{K(\beta)}-\frac{\nabla R_{\max }}{R_{\max }}\right) \frac{K(\beta)}{2 \pi^{2} R_{\max }} I_{1}-j k \frac{K(\beta) \nabla R_{\max }}{2 \pi^{2} R_{\max }} I_{2} \\
& +j k \frac{\beta^{2} \nabla K(\beta)}{2 \pi^{2}} I_{3},
\end{aligned}
$$


where

$$
\begin{gathered}
I_{1}=\int_{0}^{1} e^{-j k R_{\max } \operatorname{dn}[\xi K(\beta)]} d \xi, \\
I_{2}=\int_{0}^{1} \operatorname{dn}[\xi K(\beta)] e^{-j k R_{\max } \operatorname{dn}[\xi K(\beta)]} d \xi,
\end{gathered}
$$

and

$$
I_{3}=\int_{0}^{1} \xi \operatorname{sn}[\xi K(\beta)] \operatorname{cn}[\xi K(\beta)] e^{-j k R_{\max } \operatorname{dn}[\xi K(\beta)]} d \xi
$$

are integrals involving Jacobi elliptic functions. The integrands of (6)-(8) are analytic and hence can be integrated to any desired accuracy using, say, GaussLegendre quadrature. The Jacobi elliptic functions may be computed using algorithms in [2]. Additional terms are defined as

$$
\begin{gathered}
\nabla K(\beta)=\frac{1}{\beta}\left[\frac{E(\beta)}{1-\beta^{2}}-K(\beta)\right] \nabla \beta=\left[\frac{E(\beta)}{1-\beta^{2}}-K(\beta)\right]\left(\frac{\hat{\boldsymbol{\rho}}}{2 \rho}-\frac{\nabla R_{\max }}{R_{\max }}\right), \\
\nabla \beta=2 \nabla\left(\frac{\sqrt{\rho a}}{R_{\max }}\right)=\left(\sqrt{\frac{a}{\rho}} \frac{\hat{\boldsymbol{\rho}}}{R_{\max }}-\frac{2 \sqrt{\rho a}}{R_{\text {max }}^{2}} \nabla R_{\max }\right)=\beta\left(\frac{\hat{\boldsymbol{\rho}}}{2 \rho}-\frac{\nabla R_{\max }}{R_{\max }}\right),
\end{gathered}
$$

and

$$
\nabla R_{\max }=\frac{(\rho+a) \hat{\boldsymbol{\rho}}+\left(z-z^{\prime}\right) \hat{\mathbf{z}}}{R_{\max }} .
$$

The use of (9) allows (5) to be rewritten as

$$
\begin{aligned}
\nabla K\left(\rho, z-z^{\prime}\right)= & {\left[\frac{1}{2 \pi^{2} R_{\max }\left(1-\beta^{2}\right)}\left(\frac{\hat{\boldsymbol{\rho}}}{2 \rho}-\frac{\nabla R_{\max }}{R_{\max }}\right)\right]\left[\frac{\left(1-\beta^{2}\right)}{\beta^{2}}\left(\frac{E(\beta)}{\left(1-\beta^{2}\right)}-K(\beta)\right)\right] } \\
& \times\left[\beta^{2}\left(I_{1}+j k R_{\max } \beta^{2} K(\beta) I_{3}\right)\right]-\frac{K(\beta) \nabla R_{\max }}{2 \pi^{2} R_{\max }^{2}}\left(I_{1}+j k R_{\text {max }} I_{2}\right) .
\end{aligned}
$$

For self terms, the normal derivative is expected to have a delta function behavior as $\rho \rightarrow a+, z \rightarrow z^{\prime}$. To exhibit this, we note that the first term in brackets in (12) is the most singular and has the limiting form

$$
\nabla K\left(\rho, z-z^{\prime}\right) \underset{\rho \rightarrow a+, z \rightarrow z^{\prime}}{\longrightarrow} \frac{1}{2 \pi^{2} R_{\min }^{2}}\left[\frac{\left(z-z^{\prime}\right)^{2}+\left(a^{2}-\rho^{2}\right)}{2 \rho R_{\max }} \hat{\boldsymbol{\rho}}-\frac{\left(z-z^{\prime}\right)}{R_{\max }} \hat{\mathbf{z}}\right],
$$

where

$$
R_{\text {min }}=\sqrt{\left(z-z^{\prime}\right)^{2}+(\rho-a)^{2}} .
$$

When $\left|z-z^{\prime}\right| \ll|\rho+a|$, (13) becomes

$$
\nabla K\left(\rho, z-z^{\prime}\right) \underset{|z-z|<\rho+a \mid}{\longrightarrow}-\frac{1}{4 \pi a}\left[\frac{a(\rho-a)}{\pi \rho\left[\left(z-z^{\prime}\right)^{2}+(\rho-a)^{2}\right]}\right] \hat{\boldsymbol{\rho}}
$$

However, in the limit as $\rho$ approaches $a+$, it can be shown that the term in brackets becomes a delta function, allowing (15) to be rewritten as

$$
\nabla K\left(\rho, z-z^{\prime}\right) \underset{\left|z \rightarrow z^{\prime}\right| \ll|\rho+a|}{\longrightarrow}-\frac{1}{4 \pi a} \delta\left(z-z^{\prime}\right) \hat{\boldsymbol{\rho}} .
$$


Thus, near the source the factor (15) will dominate the field. The mid- and farrange dependence on $R$ is determined by substituting

$$
\frac{\hat{\mathbf{\rho}}}{2 \rho}-\frac{\nabla R_{\max }}{R_{\max }}=\frac{(a+\rho)(a-\rho)+\left(z-z^{\prime}\right)^{2}}{R_{\max }^{2}} \frac{\hat{\mathbf{\rho}}}{2 \rho}-\frac{\left(z-z^{\prime}\right) \hat{\mathbf{z}}}{R_{\max }^{2}}
$$

and (11) into (12) to get

$$
\begin{aligned}
\nabla K\left(\rho, z-z^{\prime}\right)= & {\left[\frac{1}{2 \pi^{2}\left(1-\beta^{2}\right)}\left(\frac{(a+\rho)(a-\rho)+\left(z-z^{\prime}\right)^{2}}{R_{\max }^{3}} \frac{\hat{\boldsymbol{\rho}}}{2 \rho}-\frac{\left(z-z^{\prime}\right) \hat{\mathbf{z}}}{R_{\max }^{3}}\right)\right] } \\
& \times\left[\frac{\left(1-\beta^{2}\right)}{\beta^{2}}\left(\frac{E(\beta)}{\left(1-\beta^{2}\right)}-K(\beta)\right)\right]\left[\beta^{2}\left(I_{1}+j k R_{\max } \beta^{2} K(\beta) I_{3}\right)\right] \\
- & \frac{K(\beta)}{2 \pi^{2}}\left(\frac{(\rho+a) \hat{\mathbf{p}}+\left(z-z^{\prime}\right) \hat{\mathbf{z}}}{R_{\max }^{3}}\right)\left(I_{1}+j k R_{\max } I_{2}\right),
\end{aligned}
$$

in which we see a common factor of $1 / R_{\max }^{3}$.

\section{Results}

The $\rho$ component of the gradient of the kernel is calculated for a $z$-directed wire segment with a radius $a$ of $1 \mathrm{~mm}$ centered about the origin. The observation points are located $0.1 \mathrm{~mm}$ from the wire surface and vary in $\Delta z=z-z^{\prime}$ from $0^{+}$to $5 a$. The results are shown in Fig. 1, where the data are compared to results calculated using the formulation in [3], which is based on the extended kernel technique. The percent difference in both the $\rho$ and $z$ components relative to the method in [3] is shown in Fig. 2.

\section{Summary and Conclusions}

A formulation of the gradient of the thin wire kernel is presented. This formulation is useful when the observation point is close to, but not on the wire. Results are compared with previous formulations to demonstrate the accuracy of the approach. This formulation is readily applied to integrations over the wire using approaches similar to those in [1].

\section{References}

[1] D. R. Wilton and N. J. Champagne, "Evaluation and integration of the thin wire kernel," IEEE Trans. Antennas Propagation, vol. 54, no. 4, pp. 1200-1206, 2006.

[2] W. H. Press, W. T. Vetterling, S A. Teukolsky, B R. Flannery, Numerical Recipes in Fortran: The Art of Scientific Computing (Second Edition), Cambridge: Cambridge University Press, 1992.

[3] N. J. Champagne, "A Method of Moments Formulation using Linear and Curved Wire Segments," Technical Report No. 95-15, Applied Electromagnetics Laboratory, Department of Electrical \& Computer Engineering, University of Houston, 1995. 


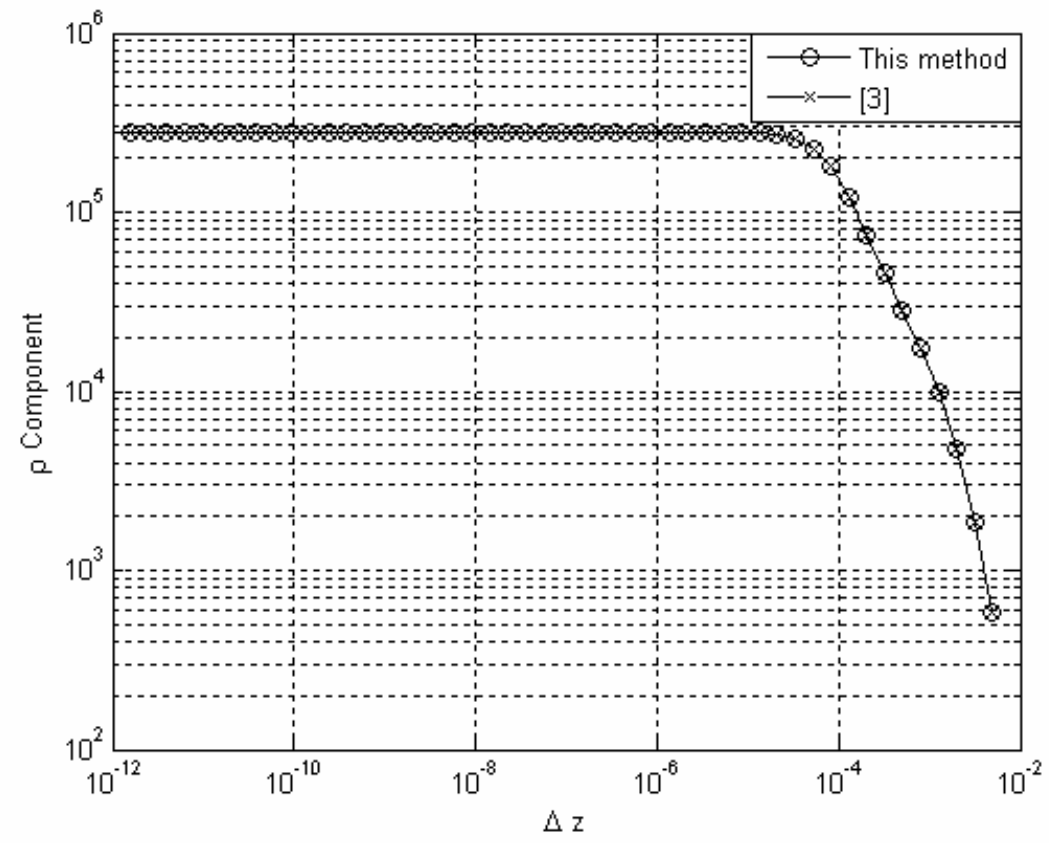

Fig. 1. The $\rho$ component of the gradient of the kernel.

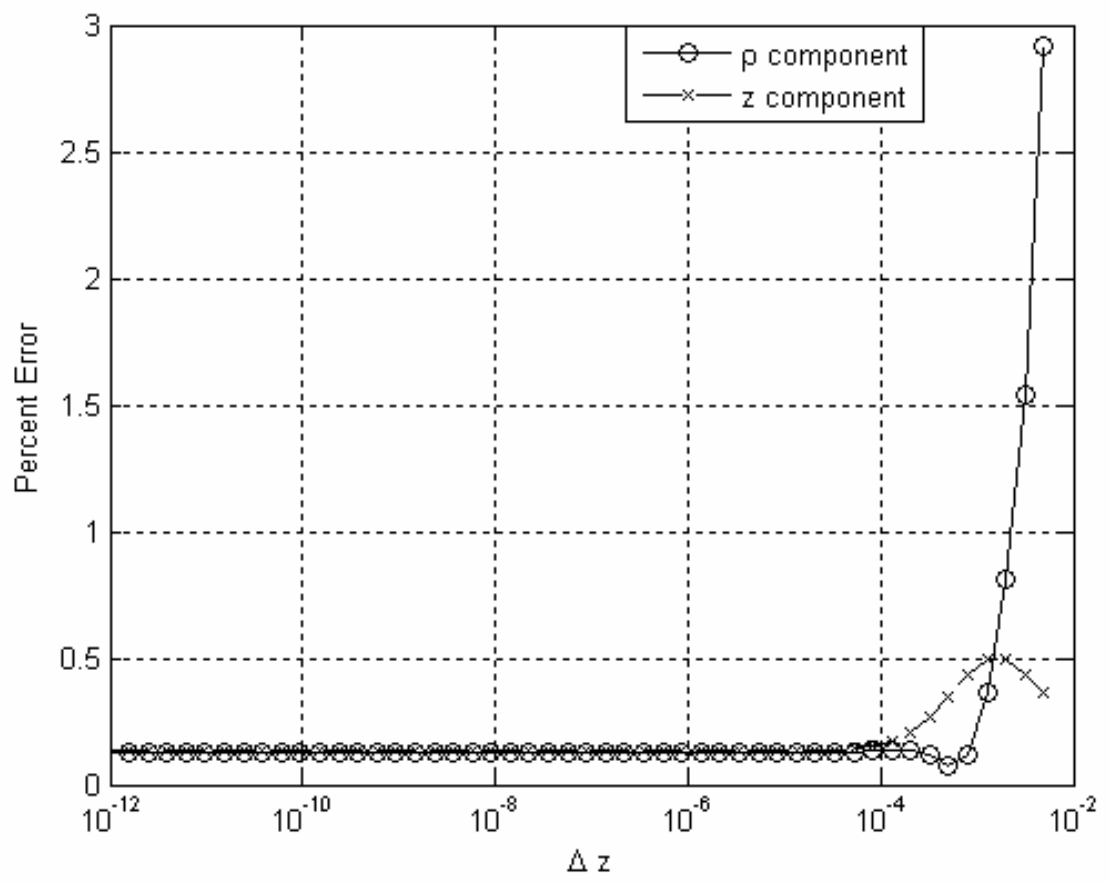

Fig. 2. The percent difference relative to the formulation in [3]. 